\title{
Un outil de cartographie, la TACF (telomere-associated chromosome fragmentation)
}

L'existence de réarrangements chromosomiques a, de longue date, aidé les chercheurs à la localisation de marqueurs et à l'établissement de cartes génétiques. Plutôt que de dépendre de la survenue aléatoire d'aberrations chromosomiques spontanées, deux techniques, les hybrides irradiés et le transfert de chromosomes en métaphase, avaient été développées dans le but d'induire des fractures chromosomiques améliorant encore l'assignation et l'agencement des marqueurs le long du chromosome. Ces approches sont parfois malheureusement associées à des délétions interstitielles ou à des liaisons artéfactuelles entre chromosome humain et chromosome de rongeur. Le développement des techniques de clonage de grands fragments d'ADN dans la levure (YAC, yeast artificial chromosome) avait révélé l'importance des séquences télomériques pour le maintien et la stabilité des chromosomes au cours de la multiplication cellulaire. Deux équipes anglaises ont récemment mis à profit cette notion pour développer un troisième moyen d'engendrer des cassures chromosomiques, soit aléatoires, soit ciblées : la TACF (telomere-associated chromosome fragmentation).

L'équipe de Goodfellow (Londres, GB) et Cooke (Edimbourg, GB) [1] a utilisé cette approche pour enrichir des hybrides cellulaires en fragments de chromosome X humain. Un vecteur linéarisé contenant un gène de sélection (le gène de résistance à l'hygromycine) et une séquence télomérique a été cellules d'hybride somatique n'ayant retenu que le chromosome $\mathrm{X}$ humain. Les cellules recombinantes sont ensuite placées dans un milieu sélectif contenant de l'hygromycine d'une part et de la 6-thioguanine d'autre part. Cette double sélection permet de ne retenir que les cellules qui ont à la fois perdu le gène HPRT (et qui deviennent par conséquent résistantes à la 6-thioguanine) et intégré le gène de l'hygromycine. Les clones cellulaires ainsi obtenus ont en effet subi une cassure du bras long du chromosome X emportant le gène HPRT humain, localisé en Xq26, suivie d'une intégration du plasmide linéarisé exogène avec sa séquence télomérique. Un nouveau chromosome, plus court, est ainsi reconstitué et reste donc stable au cours des divisions cellulaires grâce à la présence du nouveau télomère. Pour la grande majorité d'entre ces clones, ni réarrangement grossier ni délétion interstitielle ne se sont produits au site de cassure-intégration. Cependant, il semble que cet événement s'accompagne fréquemment de très larges duplications (1 à 2 mégabases) des séquences terminales jouxtant le site d'intégration du plasmide.

L'équipe de Brown et Porter (Oxford, GB) [2] a suivi la même approche, mais dans l'intention, cette fois, de cibler le point de cassure au sein d'un gène choisi en utilisant un crible grâce auquel les clones ayant subi un événement de recombinaison homologue sont sélectionnés. La fréquence était ici de 1 recombinaison homologue pour
1500 intégrations aléatoires. Ce travail fait donc la preuve de la faisabilité du ciblage du point de cassure et a en outre permis de déterminer l'orientation transcriptionnelle du gène ciblé par rapport au centromère. En conclusion, on peut envisager de nombreuses applications à cette technique TACF : il s'agit non seulement d'un outil précieux de cartographie physique des gènes, mais également d'un artifice permettant d'isoler de nouveaux marqueurs ou de cloner de nouveaux gènes. En effet, le vecteur intégré avec sa séquence télomérique peut alors servir d'hameçon pour "pêcher " la séquence d'ADN adjacente. Cela peut également constituer un moyen d'appréhender la mécanique chromosomique, c'est-à-dire d'étudier, par exemple, les séquences impliquées dans la stabilité des chromosomes au cours de la mitose.

H. G.

1. Farr CJ, Stevanovic M, Thomson EJ, Goodfellow PN, Cooke HJ. Telomere-associated chromosome fragmentation : applications in genome manipulation and analysis. Nature Genet $1992 ; 2$ : 275-82.

2. Itzhaki JE, Barnett $M$, Mac Carthy $A B$, Buckle VJ, Brown WRA, Porter ACG. Targeted breakage of a human chromosome mediated by cloned human telomeric DNA. Nature Genet $1992 ; 2: 283-7$ 